\title{
Polymers and Permeation Enhancers: Specialized Components of Mucoadhesives
}

\author{
*Amit Alexander, Ajazuddin, Swarna, Mukesh Sharma, D. K. Tripathi \\ Rungta College of Pharmaceutical Sciences and Research, Bhilai, (C.G), India.
}

\begin{abstract}
Review Article
ABSTRACT

Mucoadhesive polymers have recently gained interest among pharmaceutical scientists as a means of improving drug delivery by promoting dosage form residence time and contact time with the mucous membranes. Mucoadhesion occurs between two surfaces, one of which is a mucous membrane and another is drug delivery system. Pharmaceutical aspects of mucoadhesion have been the subject of great interest during recent years because mucoadhesion could be a solution for bioavailability problems that result from a too short length of stay of the pharmaceutical dosage form at the absorption site within the gastro-intestinal tract. It has been a great challenge to the pharmaceutical sciences in order to enhance localised drug delivery or to deliver 'difficult' molecules (proteins and oligonucleotides) into the systemic circulation. Mucoadhesive systems remain in close contact with the absorption tissue, the mucous membrane, releasing the drug at the site of action leading to increase in bioavailability (both local and systemic effects). Extending the residence time of a dosage form at a particular site and controlling the release of drug from the dosage form are useful especially for achieving controlled plasma level of the drug as well as improving bioavailability. The present review describes mucoadhesion, mucoadhesive polymers and use of these polymers in designing different types of mucoadhesive drug delivery systems.
\end{abstract}

Key words: Mucoadhesion, Mucoadhesive polymers, Mucoadhesive force, Bioadhesive property.

\section{INTRODUCTION}

Mucoadhesives are synthetic or natural polymers that interact with the mucous layer covering mucosal epithelial surface, main molecules constituting a major part of mucus (Patil et al., 2006). Mucoadhesion is a topic of current interest in the design of drug delivery systems (Asane, 2007). Mucoadhesion is the relatively new and emerging concept in drug delivery. Mucoadhesion keeps the delivery system adhering to the mucous membrane (Semalty, 2006). Mucoadhesion can be defined as the ability of synthetic or biological

\footnotetext{
*Corresponding Author

Amit Alexander

Department of Pharmaceutics

Rungta College of Pharmaceutical Sciences and

Research, Bhilai, (C.G), India.

E-mail: mailme_amitalex@yahoo.in

Contact No.: +91-9907333846
}

macromolecules to adhere to mucosal tissues. It is the ability of a material (synthetic or biological) to adhere to a biological tissue for an extended period of time to improve and enhance the bioavailability of drugs (Bhatt, 1998). In case of mucoadhesion, the biological tissue is the mucous membrane (Patil et al., 2006). The first stage involves an intimate contact between a mucoadhesive polymer and a membrane, either from good wetting of the mucoadhesive surface or from the swelling of the mucoadhesive. In the second stage, after contact is established, penetration of the mucoadhesive into the crevice of the tissue surface or interpenetration of the chains of the mucoadhesive with those of the mucous takes place. The third stage involves formation of chemical bonds between the entangled chains (Bhatt, 2009; Aidoo, 2008; Smart, 2005; Hagerstrom, 2006, Sharma et al., 2009). 
Table 1. Classification of polymers based on source.

\begin{tabular}{ll}
\hline Natural Polymers & Synthetic Polymers \\
\hline Agarose & Polymers based on poly(meth)acrylic acid. \\
Chitosan & Carbopol \\
Gelatin & Polycarbophil \\
Hyaluronic acid & Polyacrylic acid \\
Carrageenan & Polyacrylates \\
Pectin & Copolymer of acrylic acid \\
Sodium alginate. & Polyethylene glycol \\
& Copolymer of methylvinyl ether and \\
Cellulose derivatives & Methacrylic acid \\
Carboxy methyl cellulose. & Poly-2-hydroxyethylmethacrylate \\
Thiolated Carboxy methyl cellulose & Copolymer of acrylic acid and \\
Sodium Carboxy methyl cellulose & Ethylhexylacrylate \\
Hydroxyethylcellulose, & Polymethacrylate \\
Hydroxypropylcellulose, & Polyalkylcyanoacrylates \\
Hydroxypropylmethylcellulose & Polyisobutylcyanoacrylate \\
Methylcellulose & Polyisohexylcyanoacrylate. \\
Methylhydroxyethylcellulose. & \\
& Others \\
& Poly-N-2-hydroxypropylmethacrylamide \\
& Polyhydroxyethylene \\
& Poly vinyl alcohol \\
& Poly vinyl pyrrolidine \\
& Thiolated polymers \\
\hline
\end{tabular}

Table 2. Classification of polymers based on aqueous solubility.

\begin{tabular}{ll}
\hline Water Soluble Polymers & Water Insoluble Polymers \\
\hline Cellulose derivatives & Polymers based on poly(meth)acrylic acid \\
Carboxy methyl cellulose & Carbopol \\
Thiolated Carboxy methyl cellulose & Polycarbophil \\
Sodium Carboxy methyl cellulose & Polyacrylic acid \\
Hydroxyethylcellulose & Polyacrylates \\
Hydroxypropylcellulose & Copolymer of acrylic acid \\
Hydroxy propyl methyl cellulose & PEG \\
Methylcellulose & Copolymer of methylvinyl ether \\
Methylhydroxyethylcellulose. & Methacrylic acid \\
& Poly-2-hydroxyethylmethacrylate \\
Others & Copolymer of acrylic acid and \\
Poly-N-2-hydroxypropylmethacrylamide & Ethylhexylacrylate \\
Polyhydroxyethylene & Polymethacrylate \\
Poly vinyl alcohol & Polyalkylcyanoacrylates \\
Poly vinyl pyrrolidine & Polyisobutylcyanoacrylate \\
Thiolated polymers. & Polyisohexylcyanoacrylate \\
Ethylcellulose & \\
\hline
\end{tabular}


Table 3. Classification of polymers based on charge.

\begin{tabular}{lll}
\hline Cationic Polymers & Anionic Polymers & Non ionic Polymers \\
\hline Aminodextran & Carboxy methyl cellulose & Hydroxy ethyl starch \\
Chitosan & Pectin & Hydroxy propyl cellulose \\
& Cabopols & Polyethyleneglycol, \\
& Polyacrylates & Polyvinylalcohol, \\
& & Polyvinylpyrrolidine \\
& & Eudragit- NE30D \\
\hline
\end{tabular}

\section{MUCOADHESIVE POLYMERS}

Polymer is a generic term used to describe a very long molecule consisting of structural units and repeating units connected by covalent chemical bonds. The term is derived from the Greek words: polys meaning 'many' and meros meaning 'parts' (Punitha and Girish, 2010). A polymer is a substance formed by the linkage of a large number of small molecules known as monomers. Mucoadhesive polymers are watersoluble and water insoluble polymers, which have swellable networks, jointed by crosslinking agents. These polymers possess optimal polarity to make sure that they permit sufficient wetting by the mucous and optimal fluidity that permits the mutual adsorption and interpenetration of polymer and mucous takes place (Roy et al., 2006).

\section{CLASSIFICATION OF POLYMERS}

The polymers can be classified based on source (Table 1), solubility (Table 2), charge (Table 3) and bioadhesive forces (Table 4).

Depending upon source (Chickering et al., 1996; Punitha and Girish, 2010)

A. Natural Polymers

B. Synthetic Polymers

Depending upon aqueous solubility (Semalty, 2006; Roy et al., 2006)
A. Water Soluble
B. Water insoluble

Table 4. Classification of polymers based on bioadhesive forces.

\begin{tabular}{ccc}
\hline Covalent Bonds & $\begin{array}{c}\text { Electrostatic } \\
\text { Interactions }\end{array}$ & Hydrogen Bonds \\
\hline Cyanoacrylate & Chitosan & $\begin{array}{c}\text { Acrylates } \\
\text { Carbopol } \\
\text { Polycarbophil } \\
\text { Polyvinylalcohol }\end{array}$ \\
\hline
\end{tabular}

Depending upon charge (Abnawe, 2009; Majumdar et al., 2010): A. Cationic polymers

B. Anionic polymers

C. Nonionic polymers

Depending upon potential bioadhesive forces (Punitha \& Girish, 2010): A. Covalent Bonds.

B. Electrostatic Interactions.

C. Hydrogen Bonds.

Table 5. Order of mucoadhesive force for various polymers (Roy et al., 2010; Hunt et al., 1987; Abnawe, 2009).

\begin{tabular}{lc}
\hline Mucoadhesive Polymers & $\begin{array}{c}\text { Mean Adhesive Force } \\
\text { (\%) with Standard } \\
\text { Deviation }\end{array}$ \\
\hline Poly(acrylic acid) & $185.0 \pm 10.3$ \\
Tragacanth & $154.4 \pm 7.5$ \\
Poly(methylvinylether & $147.7 \pm 9.7$ \\
comaleic anhydride) & $128.6 \pm 4.0$ \\
Poly(ethylene oxide) & $128.0 \pm 2.4$ \\
Methylcellulose & $126.2 \pm 12.0$ \\
Sodium alginate & $125.2 \pm 16.7$ \\
$\begin{array}{l}\text { Hydroxypropylmethyl } \\
\text { cellulose }\end{array}$ & $125.2 \pm 4.8$ \\
Karaya gum & $117.4 \pm 4.2$ \\
Methylethyl cellulose & $117.2 \pm 3.1$ \\
Soluble starch & $115.8 \pm 5.6$ \\
Gelatin & $100.0 \pm 2.4$ \\
Pectin & $97.6 \pm 3.9$ \\
Poly (vinyl pyrrolidone) & $96.0 \pm 7.6$ \\
Poly (ethylene glycol) & $94.8 \pm 4.4$ \\
Poly ( vinyl alcohol) & $88.4 \pm 2.3$ \\
meth(hydroxyethyl- & $87.1 \pm 13.3$ \\
Hydroxypropylcellulose & \\
\hline & \\
\hline
\end{tabular}


Table 6. Relative mucoadhesive performance of some potential bio (muco) adhesive pharmaceutical polymers (Ganga, 2007; Rathore et al., 2009; Yadav et al., 2010).

\begin{tabular}{cc}
\hline Polymer & $\begin{array}{c}\text { Bioadhesive } \\
\text { Property }\end{array}$ \\
\hline Carboxy methyl cellulose & +++ \\
Hydroxy propyl methyl cellulose & ++ \\
Carbopol 934 & +++ \\
Tragacanth & +++ \\
Sodium alginate & +++ \\
Polycarbophil & +++ \\
Hydroxy ethyl cellulose & ++ \\
Gelatin & ++ \\
Guar gum & ++ \\
Gum karaya & ++ \\
Pectin & + \\
Acacia & + \\
Polyvinyl pyrrolidone & + \\
\hline
\end{tabular}

\section{PERMEATION ENHANCERS}

Substances that facilitate the permeation through mucosa are referred to as permeation enhancers. Membrane permeation is the limiting factor for many drugs in the development of mucoadhesive delivery system. The epithelium that lines the mucosa is a very effective barrier to the absorption of drugs especially buccal mucosa (Chattarajee and Walker, 1995). The efficacy of enhancer in one site is not same in the other site because of differences in cellular morphology, membrane thickness, enzymatic activity, lipid composition and potential protein interactions are structural and functional properties (Shojaei, 1998).

\section{Properties}

According to Aungst (1994) permeation enhancers should be-

- Safe

- Non- toxic

- Non -irritant

- Non-allergeic

- Pharmacologically and chemically inert

Surfactants such as anionic, cationic, nonionic and bile salts increase permeability of drugs by perturbation of intercellular lipids. Chelators act by interfering with the calcium ions. Fatty acids act by increasing fluidity of phospholipids. Positively charged polymers act by ionic interaction with negative charge on the mucosal surface (Schipper et al., 2004).

Table 7. List of permeation enhancers (Lee et al., 2000)

\begin{tabular}{|c|c|}
\hline Chelators & EDTA, Citric acid, Sodium salicylates, Methoxy salicylates \\
\hline Surfactants & $\begin{array}{l}\text { Sodium lauryl sulphate, Polyoxyethylene, Polyoxyethylene-9-laurylether , } \\
\text { Polyoxythylene-20-cetylether, Benzalkonium chloride, 23-lauryl ether, Cetylpyridinium } \\
\text { chloride, Cetyltrimethyl ammonium bromide }\end{array}$ \\
\hline Bile Salts & $\begin{array}{l}\text { Sodium glycocholate, Sodium deoxycholate, Sodium taurocholate, Sodium } \\
\text { glycodeoxycholate, Sodium taurodeoxycholate }\end{array}$ \\
\hline Fatty Acids & $\begin{array}{l}\text { Oleic acid, Capric acid, Lauric acid, Lauric acid/ propylene glycol, Methyloleate, } \\
\text { Lysophosphatidylcholine, Phosphatidylcholine }\end{array}$ \\
\hline Non Surfactants & Unsaturated cyclic ureas. \\
\hline Inclusion Complexes & Cyclodextrins \\
\hline Thiolated Polymers & $\begin{array}{l}\text { Chitosan-4-thiobutylamide, Chitosan-cysteine, Poly (acrylic acid)-homocysteine, } \\
\text { Polycarbophil-cysteine, Polycarbophil-cysteine/gsh, Chitosan-4-thioethylamide/gsh, } \\
\text { Chitosan- 4-thioglycholic acid }\end{array}$ \\
\hline Others & $\begin{array}{l}\text { Aprotinin, Azone, Cyclodextrin, Dextran sulfate, Menthol, Polysorbate } 80 \text {, Sulfoxides } \\
\text { and various alkyl glycosides. }\end{array}$ \\
\hline
\end{tabular}




\section{REFERENCES}

Abnawe SA. (2009) Mucoahesive Drug Delivery System. Pharmainfo.net. 1: 1-34

Ahuja A, Khar RK, Ali J. (1997) Mucoadhesive drug delivery systems. Drug Develop Indus Pharm. 23:489-515.

Allen C, Maysinger D, Eisenberg A. (1999) Nanoengineering block copolymer aggregates for drug delivery, Col. Surf. B: Biointerfaces. 16: 3-27.

Andrew GP, Laverty TP, Jones DS. (2009) Mucoadhesive polymeric for controlled drugdelivery. European Journal of Pharmaceutics and Biopharmaceutics 71 : 505-518.

Asane GS. (2007) Mucoadhesive Gastro Intestinal Drug Delivery System. Pharmainfo.net. 5:1-6.

Aungst A. (1994) Permeability and metabolism as barriers to transmucosal delivery of peptides and proteins, Drug Permeation Enhancement. Theory and Applications, Marcel Dekker, New York. 1: 323-343.

Benedetto DA, Shah DO, Kaufman HE. (1975) The instilled fluid dynamics and surfacechemistry of polymers in the preocular tear film. Investigative Ophthalmology. $14: 887-902$.

Bhatt JH. (2009) Designing And Evaluation of Mucoadhesive Microspheres of Metronidazole for Oral Controlled Drug Delivery. Pharmainfo.net. 2 : 1-13.

Bhatt PP, Johnston TP. ( 1998). Evaluation of a mucoadhesivebuccal patch for delivery of peptides: In vitro screening of bioadhesion. Drug DevInd Pharm. 24:19-26.

Chickering D., Jacob J., Mathiowitz E. (1996) Poly (fumariccosebacic) microspheres as oral drug delivery systems. BiotechnolBioeng. 52:96-101.

Das Bibin K., Deepa P. (2009) Mucoadhesive Agents. B.Pharm. Project and review articles. $1: 1-29$.

Hagerstrom H. (2006) Polymer gels as Pharmaceutical Dosage Forms,Comprehensive Summaries of Uppsala Dissertationsfrom the Faculty of Pharmacy 293. 1: 1-17.

Hunt G, Kearney P, Kellaway, IW. (1987) Mucoadhesive polymers in drug delivery systems. Drug Delivery System: Fundamental and Techniqes. Elis Horwood Chichester. 2:180-185.

Kast CE, Guggi D, Langoth N , Bernkop-Schnürch A. ( 2003) Pharm. Res. 20: 931-936.

Lee JW, Park JH, Robinson JR. (2000) Bioadhesive-based dosage forms: The next generation J. Pharm. Sci. 89 : 850-866.

Leitner VM, Guggi D ,Bernkop-Schnürch A. (2003) Central Eur. Symp. Pharm. Technology, Ljubljana, Slovenia. 1: $1-12$.

Punitha S, Girish Y. (2010) Polymers in mucoadhesivebuccal drug delivery system. International Journal of Research and Pharmaceutical Sciences.1:170-186.

Rajput GC, Majmudar FD, Patel JK, Patel KN, Thakor RS, Patel BP, Rajgor NB. (2010) Stomach Specific Mucoadhesivemicrosphere as a controlled drug delivery system, Systematic Reviews In Pharmacy.1:70-78.
Rathore K. (2009) Formulation and Evaluation of Mucoadhesive Drug Delivery systems. Pharma Times. 35:29-35.

Rossi S, Bonferoni MC, Ferrari F, Caramella C. (1999) Drug release and washability of mucoadhesive gels based on sodium carboxymethylcellulose and polyacrylic acid. Pharmaceutical development and technology. 4: 55-63.

Roy SK, Prabhakar B. (2010) Bioadhesive Polymeric Platforms for TransmucosalDrug Delivery Systems. Tropical Journal of Pharmaceutical Research. 9:91-104.

Saviae R, Eisenberg LLA, Maysinger D. (2003) Micellarnano containers distribute to defined cytoplasmic organelles. Tropical Journal of Science. 300: 615-618.

Schipper NGM, Varum KM, Artursson P. (2004) Chitosans as absorption enhancers for poorly absorbable drugs: influence of the molecular weight and degree of acetylation on drug transport across human intestinal epithelium (Caco-2) cells. Pharm. Res. 21: 344-353.

Schnurch AB. (2005) Thiomers: A new generation of mucoadhesive polymers. Advanced Drug Delivery Reviews. Elsevier. 57: 1569 - 1582.

Semalty A. ( 2006) Mucoadhesive Polymers. Pharmainfo. net. $4: 1-10$.

Sharma HK, Sarangi B, PradhanSiba P. (2009) Preparation and in-vitro evaluation of mucoadhesivemicrobeads containing Timolol Maleate using mucoadhesive substances of Dilleniaindica L..Arch Pharmaceutical Sciences \& Research. 1:181 -188.

Shojaei AH. (1998) Buccal mucosa as a route for systemic drug delivery: A review. J. Pharm. Pharmaceut. Sci. 1 : 15-30.

Smart JD. (2005) The basics and underlying mechanisms of mucoadhesion. Science Direct. 57:1556-1568.

Thielmann F, Naderi M, Khutoryanskiy V, Khutoryanskaya O. (2007) Mucoadhesivehydrogel films based on blends of poly(acrylic acid) and methylcellulose. Available at: http://www.aapsj.org/abstracts/ NBC_2007/NBC07-000679.PDF.

Valenta C. (2005) The use of mucoadhesive polymers in vaginal delivery. Advanced Drug Delivery Reviews. Elsevier. 57:1692 - 1712 .

Yadav VK, Gupta AB, Kumar R, Yadav JS , Kumar B. (2010) Mucoadhesive Polymers: Means of Improving the Mucoadhesive Properties of Drug Delivery System. J. Chem. Pharm. Res. 2:418-432. 